\title{
Design of Stable Circularly Symmetric Two-Dimensional GIC Digital Filters Using PLSI Polynomials
}

\author{
Ezra Morris Abraham Gnanamuthu, ${ }^{1}$ C. Eswaran, ${ }^{2}$ and K. Ramar ${ }^{1}$ \\ ${ }^{1}$ Faculty of Engineering, Multimedia University, Cyberjaya 63100, Selangor, Malaysia \\ ${ }^{2}$ Faculty of Information Technology, Multimedia University, Cyberjaya 63100, Selangor, Malaysia
}

Received 5 December 2005; Accepted 24 May 2007

Recommended by Ricardo Merched

A method for designing stable circularly symmetric two-dimensional digital filters is presented. Two-dimensional discrete transfer functions of the rotated filters are obtained from stable one-dimensional analog-filter transfer functions by performing rotation and then applying the double bilinear transformation. The resulting filters which may be unstable due to the presence of nonessential singularities of the second kind are stabilized by using planar least-square inverse polynomials. The stabilized rotated filters are then realized by using the concept of generalized immittance converter. The proposed method is simple and straight forward and it yields stable digital filter structures possessing many salient features such as low noise, low sensitivity, regularity, and modularity which are attractive for VLSI implementation.

Copyright (C) 2007 Ezra Morris Abraham Gnanamuthu et al. This is an open access article distributed under the Creative Commons Attribution License, which permits unrestricted use, distribution, and reproduction in any medium, provided the original work is properly cited.

\section{INTRODUCTION}

Two-dimensional (2D) digital filters find applications in many areas such as geophysics, robotics, biomedicine, image processing, and prospecting for oil $[1,2]$. A special class of $2 \mathrm{D}$ infinite impulse response (IIR) digital filters whose magnitude responses are approximately circularly symmetric can be realized by cascading a number of elementary filters known as rotated filters $[3,4]$. A rotated filter is designed by rotating a stable $1 \mathrm{D}$ analog filter and then using the double bilinear transformation to obtain the corresponding digital filter. However, the stability of these rotated digital filters is not guaranteed due to the presence of nonessential singularities of the second kind $[5,6]$. To overcome this problem, a new type of rotated filters known as pseudorotated filters has been proposed in [7]. Methods for realizing rotated and pseudorotated digital filters by using the concept of generalized immittance converter (GIC) have been reported in $[8,9]$.

In this paper, a new method is proposed for realizing stable 2D rotated GIC digital filters using planar least-square inverse (PLSI) polynomials [10-14]. It is shown [10-14] that an unstable 2D IIR digital filter can be stabilized by replacing its denominator polynomial, say $B\left(z_{1}, z_{2}\right)$, by a new polynomial $B^{\prime \prime}\left(z_{1}, z_{2}\right)$ which is the double PLSI polynomial of
$B\left(z_{1}, z_{2}\right)$ and the magnitude response of the resulting stable filter would be approximately equal to that of the original unstable filter. Though this approach is not valid for a general $2 \mathrm{D}$ polynomial, it is shown in this paper that the denominator polynomials of the $2 \mathrm{D}$ discrete transfer functions of the rotated filters belong to a specific class of $2 \mathrm{D}$ polynomials for which the PLSI-based stabilization method can be applied.

\section{ROTATED FILTERS}

Consider a stable 1D analog filter transfer function of the form

$$
\begin{aligned}
H(s) & =A_{0} \prod H_{1}(s) \prod H_{2}(s) \\
& =A_{0}\left[\prod_{j=1}^{m} \frac{s+a_{j}}{s+b_{j}}\right]\left[\prod_{j=1}^{n} \frac{s^{2}+d_{j} s+f_{j}}{s^{2}+g_{j} s+e_{j}}\right],
\end{aligned}
$$

where $a_{j}, b_{j}, d_{j}, f_{j}, g_{j}$, and $e_{j}$ are real constants.

The discrete transfer functions $H_{1}\left(z_{1}, z_{2}\right)$ and $H_{2}\left(z_{1}, z_{2}\right)$ corresponding to filters represented by $H_{1}(s)$ and $H_{2}(s)$ can be obtained by performing rotation with $s=c_{1} s_{1}+c_{2} s_{2}$, where $c_{1}$ and $c_{2}$ are real positive constants and then applying the double bilinear transformation of [4]

$$
s_{i}=\frac{2}{T_{i}} \frac{1-z_{i}}{1+z_{i}} \quad \text { for } i=1,2 .
$$


The general forms of $H_{1}\left(z_{1}, z_{2}\right)$ and $H_{2}\left(z_{1}, z_{2}\right)$ obtained by using the above procedure will be as shown:

$$
H_{1}\left(z_{1}, z_{2}\right)=\frac{N_{1}\left(z_{1}, z_{2}\right)}{D_{1}\left(z_{1}, z_{2}\right)},
$$

where

$$
\begin{gathered}
N_{1}\left(z_{1}, z_{2}\right)=\left[\begin{array}{ll}
1 & z_{1}
\end{array}\right]\left[\begin{array}{ll}
\alpha_{00} & \alpha_{01} \\
\alpha_{10} & \alpha_{11}
\end{array}\right]\left[\begin{array}{c}
1 \\
z_{2}
\end{array}\right], \\
D_{1}\left(z_{1}, z_{2}\right)=\left[\begin{array}{ll}
1 & z_{1}
\end{array}\right]\left[\begin{array}{ll}
\beta_{00} & \beta_{01} \\
\beta_{10} & \beta_{11}
\end{array}\right]\left[\begin{array}{c}
1 \\
z_{2}
\end{array}\right] \\
H_{2}\left(z_{1}, z_{2}\right)=\frac{N_{2}\left(z_{1}, z_{2}\right)}{D_{2}\left(z_{1}, z_{2}\right)},
\end{gathered}
$$

where

$$
\begin{aligned}
& N_{2}\left(z_{1}, z_{2}\right)=\left[\begin{array}{lll}
1 & z_{1} & z_{1}^{2}
\end{array}\right]\left[\begin{array}{lll}
\alpha_{00} & \alpha_{01} & \alpha_{02} \\
\alpha_{10} & \alpha_{11} & \alpha_{12} \\
\alpha_{20} & \alpha_{21} & \alpha_{22}
\end{array}\right]\left[\begin{array}{c}
1 \\
z_{2} \\
z_{2}^{2}
\end{array}\right], \\
& D_{2}\left(z_{1}, z_{2}\right)=\left[\begin{array}{lll}
1 & z_{1} & z_{1}^{2}
\end{array}\right]\left[\begin{array}{lll}
\beta_{00} & \beta_{01} & \beta_{02} \\
\beta_{10} & \beta_{11} & \beta_{12} \\
\beta_{20} & \beta_{21} & \beta_{22}
\end{array}\right]\left[\begin{array}{c}
1 \\
z_{2} \\
z_{2}^{2}
\end{array}\right] .
\end{aligned}
$$

$\alpha_{i i}$ and $\beta_{i i}$ in (3) represent real constants.

Though the analog filter transfer functions of (1) are stable, the discrete transfer functions $H_{1}\left(z_{1}, z_{2}\right)$ and $H_{2}\left(z_{1}, z_{2}\right)$ of (3) can become unstable if nonessential singularities of the second kind are present $[5,6]$. Hence it is necessary to stabilize these transfer functions prior to their realization. For this purpose, we use an approach based on PLSI polynomials.

\section{USE OF PLSI POLYNOMIALS FOR STABILIZATION}

Shanks et al. [10] have proposed a technique based on PLSI polynomials to stabilize $2 \mathrm{D}$ recursive filters in such a way that the magnitude responses of the stabilized filters are approximately equal to those of the original unstable filters. This technique is based on the conjecture that the PLSI polynomial of an arbitrary unstable 2D polynomial is stable. Thus if $D\left(z_{1}, z_{2}\right)$ is the denominator polynomial of an unstable filter, by replacing it with the double PLSI polynomial of $D\left(z_{1}, z_{2}\right)$, we would obtain a stable filter with magnitude response close to that of the original unstable filter [10]. Shanks's conjecture has not yet been proved for a general case [11-14]. However, this conjecture has been proved for specific polynomials of the types $D_{1}\left(z_{1}, z_{2}\right)$ and $D_{2}\left(z_{1}, z_{2}\right)$ of $(3)[13,14]$.

Let $D_{1}^{\prime}\left(z_{1}, z_{2}\right)$ and $D_{2}^{\prime}\left(z_{1}, z_{2}\right)$ be the PLSI polynomials of $D_{1}\left(z_{1}, z_{2}\right)$ and $D_{2}\left(z_{1}, z_{2}\right)$ of $(3 \mathrm{c})$ and (3f), respectively. Let us assume that $D_{1}^{\prime}\left(z_{1}, z_{2}\right)$ and $D_{2}^{\prime}\left(z_{1}, z_{2}\right)$ also have the same forms as the original polynomials $D_{1}\left(z_{1}, z_{2}\right)$ and $D_{2}\left(z_{1}, z_{2}\right)$, respectively. In other words, we assume that (3c) and (3f) with different sets of coefficients, say $\beta_{i i}^{\prime}$, can represent the PLSI polynomials $D_{1}^{\prime}\left(z_{1}, z_{2}\right)$ and $D_{2}^{\prime}\left(z_{1}, z_{2}\right)$, respectively.

The coefficients $\beta_{i i}^{\prime}$ of these PLSI polynomials can be obtained from the coefficients $\beta_{i i}$ of the original polynomials by solving a matrix equation of the form $[13,14]$

$$
\Gamma \beta_{i i}^{\prime}=\beta_{i i}
$$

where $\Gamma$ is a centrosymmetric matrix whose elements represent the autocorrelation coefficients of the original polynomial.

For obtaining the coefficients $\beta_{i i}^{\prime}$ of the first-order PLSI polynomial $D_{1}^{\prime}\left(z_{1}, z_{2}\right)$, (4) takes the form $[13,14]$

$$
\left[\begin{array}{llll}
r_{0} & r_{1} & r_{3} & r_{4} \\
r_{1} & r_{0} & r_{2} & r_{3} \\
r_{3} & r_{2} & r_{0} & r_{1} \\
r_{4} & r_{3} & r_{1} & r_{0}
\end{array}\right]\left[\begin{array}{l}
\beta_{00}^{\prime} \\
\beta_{01}^{\prime} \\
\beta_{10}^{\prime} \\
\beta_{11}^{\prime}
\end{array}\right]=\left[\begin{array}{c}
\beta_{00} \\
0 \\
0 \\
0
\end{array}\right],
$$

where $r_{i}$ are the autocorrelation coefficients of the polynomial $D_{1}\left(z_{1}, z_{2}\right)$ given by

$$
\begin{aligned}
& r_{0}=\beta_{00}^{2}+\beta_{01}^{2}+\beta_{10}^{2}+\beta_{11}^{2}, \\
& r_{1}=\beta_{00} \beta_{01}+\beta_{10} \beta_{11}, \\
& r_{2}=\beta_{01} \beta_{10} \\
& r_{3}=\beta_{00} \beta_{10}+\beta_{01} \beta_{11}, \\
& r_{4}=\beta_{00} \beta_{11} .
\end{aligned}
$$

Similarly for obtaining the coefficients $\beta_{i i}^{\prime}$ of the second-order PLSI polynomial $D_{2}^{\prime}\left(z_{1}, z_{2}\right)$, we need to solve the following equation $[13,14]$ :

$$
\left[\begin{array}{lllllllll}
r_{0} & r_{1} & r_{2} & r_{5} & r_{6} & r_{7} & r_{10} & r_{11} & r_{12} \\
r_{1} & r_{0} & r_{1} & r_{4} & r_{5} & r_{6} & r_{9} & r_{10} & r_{11} \\
r_{2} & r_{1} & r_{0} & r_{3} & r_{4} & r_{5} & r_{8} & r_{9} & r_{10} \\
r_{5} & r_{4} & r_{3} & r_{0} & r_{1} & r_{2} & r_{5} & r_{6} & r_{7} \\
r_{6} & r_{5} & r_{4} & r_{1} & r_{0} & r_{1} & r_{4} & r_{5} & r_{6} \\
r_{7} & r_{6} & r_{5} & r_{2} & r_{1} & r_{0} & r_{3} & r_{4} & r_{5} \\
r_{10} & r_{9} & r_{8} & r_{5} & r_{4} & r_{3} & r_{0} & r_{1} & r_{2} \\
r_{11} & r_{10} & r_{9} & r_{6} & r_{5} & r_{4} & r_{1} & r_{0} & r_{1} \\
r_{12} & r_{11} & r_{10} & r_{7} & r_{6} & r_{5} & r_{2} & r_{1} & r_{0}
\end{array}\right]\left[\begin{array}{l}
\beta_{00}^{\prime} \\
\beta_{01}^{\prime} \\
\beta_{02}^{\prime} \\
\beta_{10}^{\prime} \\
\beta_{11}^{\prime} \\
\beta_{12}^{\prime} \\
\beta_{20}^{\prime} \\
\beta_{21}^{\prime} \\
\beta_{22}^{\prime}
\end{array}\right]=\left[\begin{array}{c}
\beta_{00} \\
0 \\
0 \\
0 \\
0 \\
0 \\
0 \\
0 \\
0
\end{array}\right],
$$

where $r_{i}$ are the autocorrelation coefficients of $D_{2}\left(z_{1}, z_{2}\right)$.

The transfer functions of the rotated filters are stabilized by replacing the denominator polynomials $D_{1}\left(z_{1}, z_{2}\right)$ and $D_{2}\left(z_{1}, z_{2}\right)$ by their double PLSI polynomials $D_{1 s}\left(z_{1}, z_{2}\right)$ and $D_{2 s}\left(z_{1}, z_{2}\right)$, respectively. The coefficients of $D_{1 s}\left(z_{1}, z_{2}\right)$ and $D_{2 s}\left(z_{1}, z_{2}\right)$ can be obtained from those of $D_{1}\left(z_{1}, z_{2}\right)$ and $D_{2}\left(z_{1}, z_{2}\right)$, respectively, by going through a two-step procedure given below.

(i) Determine the coefficients of $D_{1}^{\prime}\left(z_{1}, z_{2}\right)$ and $D_{2}^{\prime}\left(z_{1}, z_{2}\right)$ from those of $D_{1}\left(z_{1}, z_{2}\right)$ and $D_{2}\left(z_{1}, z_{2}\right)$ by applying $(5)$ and (6), respectively.

(ii) Determine the coefficients of $D_{1 s}\left(z_{1}, z_{2}\right)$ and $D_{2 s}\left(z_{1}, z_{2}\right)$ from those of $D_{1}^{\prime}\left(z_{1}, z_{2}\right)$ and $D_{2}^{\prime}\left(z_{1}, z_{2}\right)$ by applying (5) and (6), respectively, for the second time.

At the end of step (ii), we obtain the stabilized transfer functions of the rotated filters which are shown in (7) and (9):

$$
H_{1 s}\left(z_{1}, z_{2}\right)=\frac{N_{1}\left(z_{1}, z_{2}\right)}{D_{1 s}\left(z_{1}, z_{2}\right)},
$$




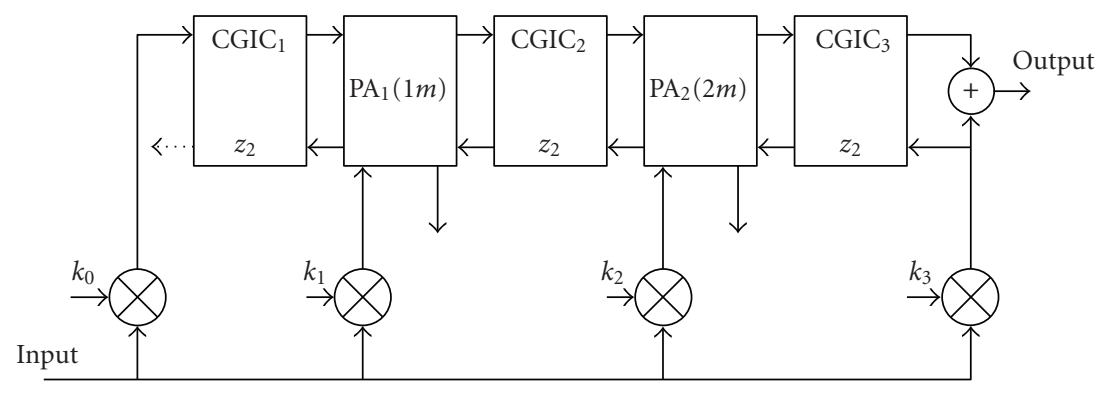

FIGURE 1: First-order CGIC-PA rotated digital filter structure.

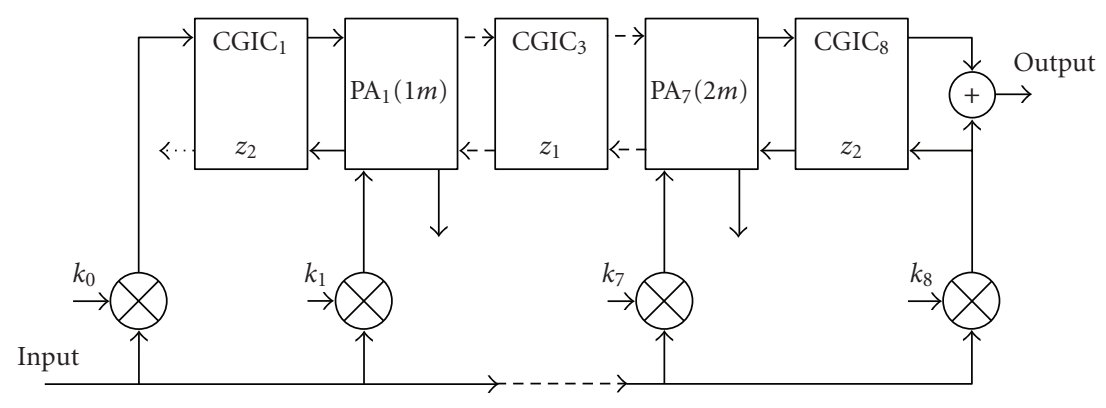

FIgURE 2: Second-order CGIC-PA rotated digital filter structure.

where

$$
\begin{gathered}
N_{1}\left(z_{1}, z_{2}\right)=\alpha_{00}+\alpha_{01} z_{2}+\alpha_{10} z_{1}+\alpha_{11} z_{1} z_{2}, \\
D_{1 s}\left(z_{1}, z_{2}\right)=\beta_{00}^{\prime \prime}+\beta_{01}^{\prime \prime} z_{2}+\beta_{10}^{\prime \prime} z_{1}+\beta_{11}^{\prime \prime} z_{1} z_{2}, \\
H_{2 s}\left(z_{1}, z_{2}\right)=\frac{N_{2}\left(z_{1}, z_{2}\right)}{D_{2 s}\left(z_{1}, z_{2}\right)}
\end{gathered}
$$

where

$$
\begin{aligned}
N_{2}\left(z_{1}, z_{2}\right)= & \alpha_{00}+\alpha_{01} z_{2}+\alpha_{02} z_{2}^{2}+\alpha_{10} z_{1}+\alpha_{11} z_{1} z_{2} \\
& +\alpha_{12} z_{1} z_{2}^{2}+\alpha_{20} z_{1}^{2}+\alpha_{21} z_{1}^{2} z_{2}+\alpha_{22} z_{1}^{2} z_{2}^{2} \\
D_{2 s}\left(z_{1}, z_{2}\right)= & \beta_{00}^{\prime \prime}+\beta_{01}^{\prime \prime} z_{2}+\beta_{02}^{\prime \prime} z_{2}^{2}+\beta_{10}^{\prime \prime} z_{1}+\beta_{11}^{\prime \prime} z_{1} z_{2} \\
& +\beta_{12}^{\prime \prime} z_{1} z_{2}^{2}+\beta_{20}^{\prime \prime} z_{1}^{2}+\beta_{21}^{\prime \prime} z_{1}^{2} z_{2}+\beta_{22}^{\prime \prime} z_{1}^{2} z_{2}^{2} .
\end{aligned}
$$

As illustrated in [10], when the PLSI technique is applied to the unstable filter, the poles are moved away from the unit bidisk, thus removing the nonessential singularity of the second kind if it is present. This concept holds good for filters of any order.

\section{REALIZATION OF ROTATED FILTERS USING GIC CONCEPT}

It is known that the $1 \mathrm{D}$ and MD digital filters designed by using the concept of GIC have many salient features such as low noise, low sensitivity, modularity, and absence of limit cycles $[8,9,15,16]$. The GIC digital filters are basically wave digital filters derived from reference analog configurations comprising GICs and resistors [15]. The method reported in $[9,16]$ can be used to realize 2D GIC digital filters directly from the given discrete transfer functions without the need for obtaining the analog reference configurations. The GIC digital filter structures which realize the transfer functions of (7) and (9) are shown in Figures 1 and 2, respectively, [9]. These filter structures make use of two types of modules known as current conversion GIC and parallel adaptors $[9,15,16]$. The multiplier constants $m_{v}$ and $k_{v}$ of Figures 1 and 2 can be determined by using the procedure reported in $[9,16]$.

\section{EXAMPLE}

Consider a first-order stable analog filter

$$
H_{1}(s)=\frac{1}{s+0.3048} .
$$

Applying the transformation $s=c_{1} s_{1}+c_{2} s_{2}$ with $c_{1}=0.1564$ and $c_{2}=0.9877$ in (11), we get

$$
H_{1}\left(s_{1}, s_{2}\right)=\frac{1}{0.1564 s_{1}+0.9877 s_{2}+0.3048} .
$$

Applying the double bilinear transformation of (2) with $T_{1}=T_{2}=1 s$, we get

$$
H_{1}\left(z_{1}, z_{2}\right)=\frac{N_{1}\left(z_{1}, z_{2}\right)}{D_{1}\left(z_{1}, z_{2}\right)}
$$

where

$$
\begin{aligned}
& N_{1}\left(z_{1}, z_{2}\right)=1+z_{1}+z_{2}+z_{1} z_{2}, \\
D_{1}\left(z_{1}, z_{2}\right)= & 2.5930-1.3577 z_{1}+0.3048 z_{2}-1.9834 z_{1} z_{2} .
\end{aligned}
$$




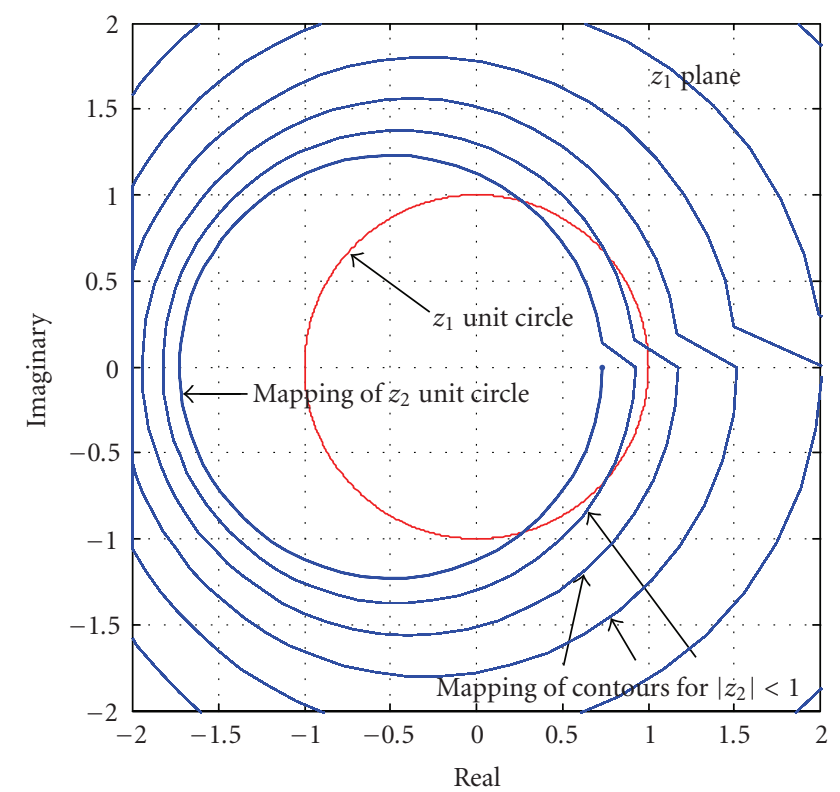

FIGURE 3: Mapping of $z_{2}$ unit circle onto $z_{1}$ plane for the unstable filter.

By using the stability conditions of a first-order 2D polynomial given in [10], we conclude that $H_{1}\left(z_{1}, z_{2}\right)$ of $(13)$ is unstable. Replacing $D_{1}\left(z_{1}, z_{2}\right)$ of (13) by its double PLSI polynomial, we get

$$
H_{1 s}\left(z_{1}, z_{2}\right)=\frac{N_{1}\left(z_{1}, z_{2}\right)}{D_{1 s}\left(z_{1}, z_{2}\right)},
$$

where

$D_{1 s}\left(z_{1}, z_{2}\right)=\left(-1.3074+0.2434 z_{2}-0.1710 z_{1}+0.3671 z_{1} z_{2}\right)$.

$H_{1 s}\left(z_{1}, z_{2}\right)$ represents a stable filter as it satisfies the stability conditions given in [10]. The stability of the 2D transfer functions of (13) and (16) can also be verified by mapping the $z_{2}$ unit circle onto $z_{1}$ plane to check whether the following theorem is satisfied [10].

Theorem 1. Given that $B\left(z_{1}, z_{2}\right)$ is a polynomial in $\left(z_{1}, z_{2}\right)$, for the coefficients of expansion of $1 / B\left(z_{1}, z_{2}\right)$ in a positive powers of $z_{1}$ and $z_{2}$ to converge absolutely, it is necessary and sufficient that $B\left(z_{1}, z_{2}\right)$ is not zero for $\left|z_{1}\right|$ and $\left|z_{2}\right|$ simultaneously less than or equal to one.

Using the procedure described in [10], the contour plots of the transfer functions are drawn as shown in Figures 3 and 4 , where the $z_{2}$ unit circle is mapped onto the $z_{1}$ plane. We note from Figures 3 and 4 that for the unstable filter, the $z_{1}$ and $z_{2}$ unit circles intersect whereas for the stable filter, they do not [10]. The normalized magnitudes of $H_{1}\left(e^{j \omega_{1}}, e^{j \omega_{2}}\right)$ and $H_{1 s}\left(e^{j \omega_{1}}, e^{j \omega_{2}}\right)$ are shown in Figures 5 and 6 , respectively. Figure 7 shows the absolute value of the error $|\varepsilon|$ between the magnitudes of $H_{1}\left(e^{j \omega_{1}}, e^{j \omega_{2}}\right)$ and $H_{1 s}\left(e^{j \omega_{1}}, e^{j \omega_{2}}\right)$. The GIC filter realizing $H_{1 s}\left(z_{1}, z_{2}\right)$ is obtained by using the procedure given in $[9,16]$.

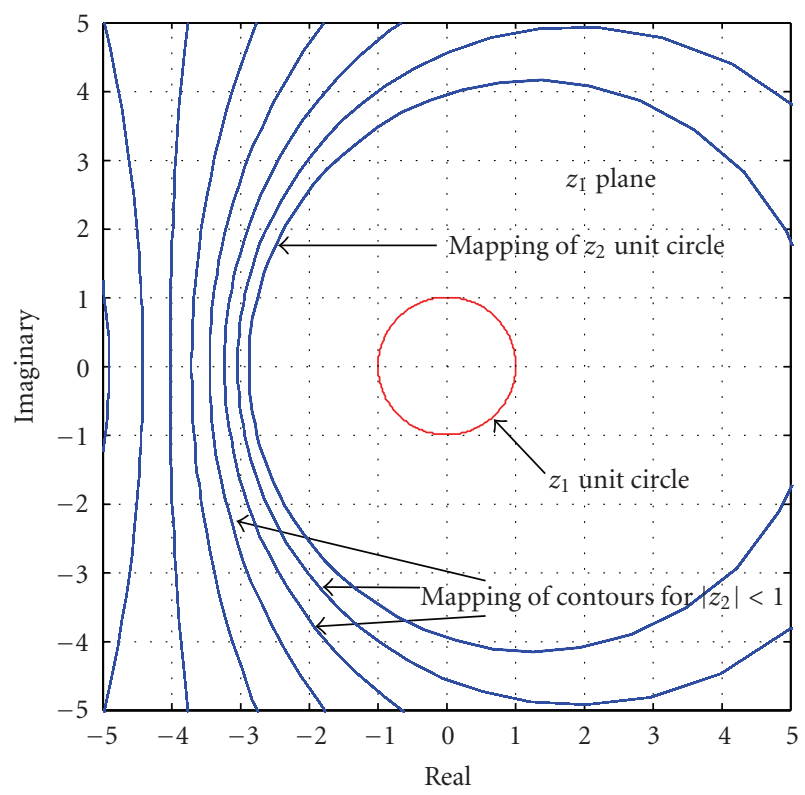

FIGURE 4: Mapping of $z_{2}$ unit circle onto $z_{1}$ plane for the stable filter.

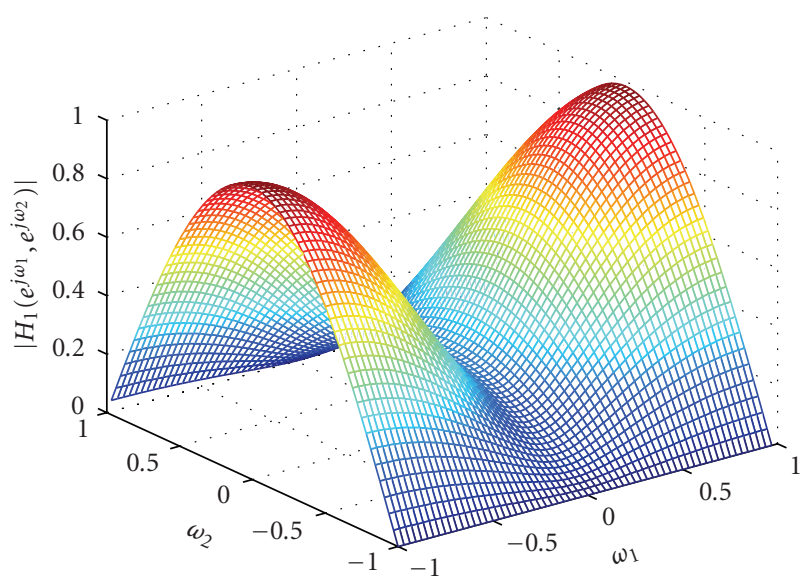

Figure 5: Normalized magnitude $H_{1}\left(e^{j \omega_{1}}, e^{j \omega_{2}}\right)$.

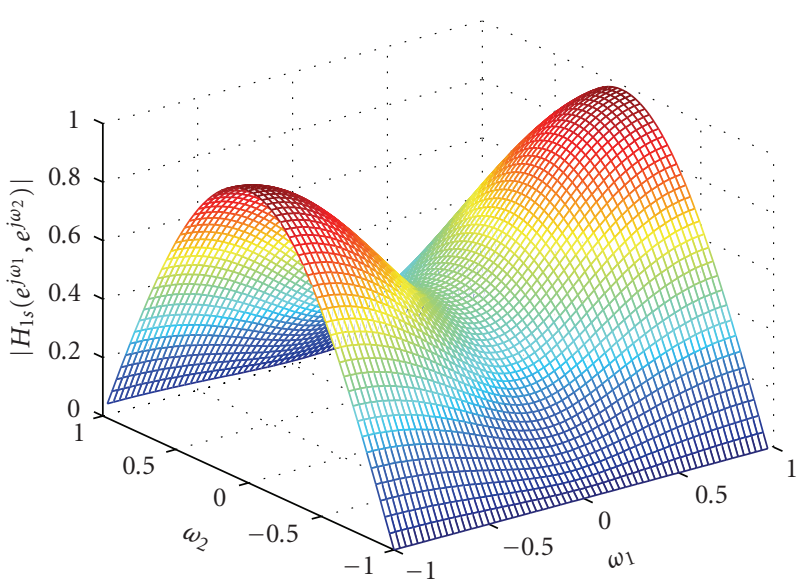

FIgURE 6: Normalized magnitude $H_{1 s}\left(e^{j \omega_{1}}, e^{j \omega_{2}}\right)$. 


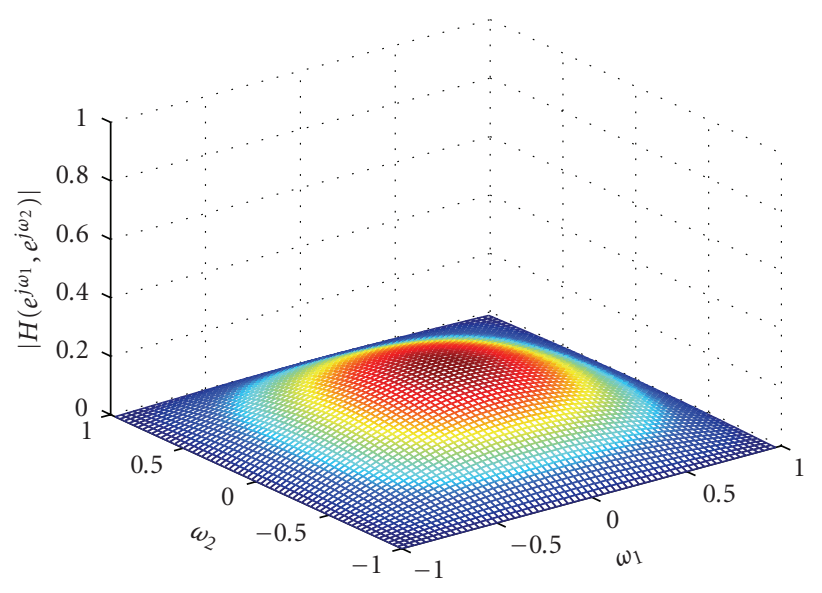

FIgURE 7: Plot of the error in magnitude response.

\section{CONCLUSIONS}

A method for realizing stable circularly symmetric 2D GIC digital filters has been presented. The proposed method makes use of PLSI polynomials for stabilizing the rotated filters. This procedure enables one to stabilize filters obtained with magnitude characteristics close to those of the original unstable filters. The steps involved in the stabilization and implementation of the rotated GIC filters can be carried out using a computer program. Since the GIC filter structures possess regularity and modularity, they are considered to be attractive for VLSI implementation.

\section{REFERENCES}

[1] K. R. Castleman, Digital Image Processing, Prentice-Hall, Englewood Cliffs, NJ, USA, 1996.

[2] R. A. Stein and N. R. Bartley, "Continuously time-variable recursive digital band-pass filters for seismic signal processing," Geophysics, vol. 48, no. 6, pp. 702-712, 1983.

[3] J. M. Costa and A. N. Venetsanopoulos, "Design of circularly symmetric two-dimensional recursive filters," IEEE Transactions on Acoustics, Speech, and Signal Processing, vol. 22, no. 6, pp. 432-443, 1974.

[4] D. M. Goodman, "A design technique for circularly symmetric low-pass filters," IEEE Transactions on Acoustics, Speech, and Signal Processing, vol. 26, no. 4, pp. 290-304, 1978.

[5] D. Goodman, "Some stability properties of two-dimensional linear shift-invariant digital filters," IEEE Transactions on Circuits and Systems, vol. 24, no. 4, pp. 201-208, 1977.

[6] P. K. Rajan, H. C. Reddy, M. N. S. Swamy, and V. Ramachandran, "Generation of two-dimensional digital functions without non-essential singularities of the second kind," IEEE Transactions on Acoustics, Speech, and Signal Processing, vol. 28, no. 2, pp. 216-223, 1980.

[7] G. V. Mendonca, A. Antoniou, and A. Venetsanopoulos, "Design of two-dimensional pseudorotated digital filters satisfying prescribed specifications," IEEE Transactions on Circuits and Systems, vol. 34, no. 1, pp. 1-10, 1987.

[8] T. Venkateswarlu and C. Eswaran, "Design of 2-D pseudorotated digital filters with multiple ouputs," IEEE Transactions on Circuits and Systems II, vol. 46, no. 2, pp. 177-179, 1999.
[9] K. Rameshwaran and C. Eswaran, "Design of two-dimensional pseudorotated digital filters using concept of generalized immittance converter," Circuits, Systems, and Signal Processing, vol. 22, no. 4, pp. 367-376, 2003.

[10] J. L. Shanks, S. Treitel, and J. Justice, "Stability and synthesis of two-dimensional recursive filters," IEEE Transactions on Audio and Electroacoustics, vol. 20, no. 2, pp. 115-128, 1972.

[11] P. S. Reddy, D. R. R. Reddy, and M. N. S. Swamy, "Proof of a modified form of Shanks' conjecture on the stability of 2-D planar least square inverse polynomials and its implications," IEEE Transactions on Circuits and Systems, vol. 31, no. 12, pp. 1009-1015, 1984.

[12] P. Delsarte, Y. Genin, and Y. Kemp, "Comments on "proof of a modified form of Shanks' conjecture on the stability of 2-D planar least square inverse polynomials and its implications",' IEEE Transactions on Circuits and Systems, vol. 32, no. 9, pp. 966-966, 1985.

[13] B. D. O. Anderson and E. I. Jury, "Proof of a special case of Shanks' conjecture," IEEE Transactions on Acoustics, Speech, and Signal Processing, vol. 24, no. 6, pp. 574-575, 1976.

[14] E. M. A. Gnanamuthu and P. S. Reddy, "The PLSI method of stabilizing 2-D recursive digital filters-a complete solution," Asian Journal of Information Technology, vol. 3, pp. 628-641, 2004.

[15] A. Antoniou, Digital Signal Processing: Signals, Systems and Filters, McGraw-Hill, New York, NY, USA, 2005.

[16] C. Eswaran and K. Rameshwaran, "A new realization method for multidimensional GIC digital filters," IEEE Transactions on Circuits and Systems I, vol. 49, no. 1, pp. 66-69, 2002.

Ezra Morris Abraham Gnanamuthu received his B.Eng. degree from Bharathiar University, India, and M.Eng. degree from Anna University, India, in 1991 and 1993, respectively. He has over 14 years of experience in the field of engineering education. He has worked with various colleges and universities in India and Malaysia. Currently, he is a research scholar with Multimedia University, Malaysia. He has published around 17 papers in leading international conferences and journals. His research interests include digital signal processing and mobile communication. He has supervised one M.S. by research student in the field of mobile communication. He is a Senior Member of IEEE.

C. Eswaran received his B.Tech., M.Tech., and Ph.D. degrees from the Indian Institute of Technology Madras, India, where he worked as a Professor in the Department of Electrical Engineering until January 2002. Currently, he is working as a Professor in the Faculty of Information Technology, Multimedia University, Malaysia. He is also serving as the Chairman for the center of excellence, Multimedia Computing, at

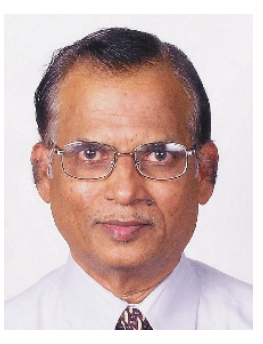
this university. Dr. Eswaran was a Research Fellow/Visiting Faculty in Ruhr University Bochum, Germany, Concordia University, Canada, University of Victoria, Canada, and Nanyang Technological University, Singapore. Dr. Eswaran has supervised successfully more than twenty Ph.D./M.S. students in the areas of digital signal processing, communications, neural networks, information systems, and biomedical engineering. He has published more than 
120 research papers in these areas in reputed international journals and conferences. He has also carried out several sponsored research projects and served as industrial consultant in these areas. Dr. Eswaran is a Senior Member of IEEE.

K. Ramar received the B.Eng. degree from University of Madras in 1964, and M.Tech. and Ph.D. degrees from Indian Institute of Technology Madras, India, in 1968 and 1972, respectively. He worked as a Professor in the Department of Electrical Engineering, Indian Institute of Technology Madras, until December 2002. He has more than 40 years of teaching and research experience. $\mathrm{He}$ is currently a Professor in the Faculty

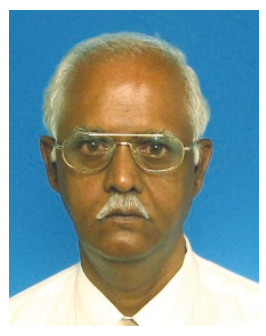
of Engineering, Multimedia University, Malaysia. He has published more than 60 papers in leading international journals and conferences. His fields of interest include power system operation and control, computer application to power systems, power systems protection, and fault location in overhead transmission lines. He is a Senior Member of IEEE. 